\section{Accidente cerebrovascular embólico secundario a fibroelastoma papilar de válvula mitral}

\author{
ROBERTO GONZÁLEZ L. ${ }^{1,2}$, RODRIGO REYES M. ${ }^{1,2}$, \\ ALEJANDRA RIQUELME U. ${ }^{2}$, ENRIQUE SEGUEL S. ${ }^{1,2}$, \\ ALECK STOCKINS L. ${ }^{1,2}$, ANDRÉS JADUE T. ${ }^{1}$, EMILIO ALARCÓN C. ${ }^{1,2}$
}

\section{Embolic stroke secondary to a mitral valve fibroelastoma. Case report}

Embolic stroke secondary to cardiac tumors is uncommon. However, 25-30\% of cardiac tumors may cause systemic emboli. We report a 29-year-old male consulting for a sudden episode of aphasia and right hemiparesis, compatible with infarct of the left middle cerebral artery territory. Transthoracic echocardiography reported an ovoid tumor of $8 \times 7 \times 7 \mathrm{~mm}$ in relation to the sub valvular apparatus of the mitral valve. After neurologic stabilization, surgical treatment was performed. Approached by median sternotomy and in cardiopulmonary bypass, the mitral valve was explored. A macroscopic tumor consistent with a papillary fibroelastoma curled in sub valvular chordae was found. It was deployed and resected from its base, while the anterior mitral leaflet was preserved intact. Histopathological examination confirmed the intraoperative macroscopic diagnosis. The patient recovered uneventfully postoperatively and was discharged on the fifth day after surgery. He currently is in functional capacity I without cardiovascular symptoms at five years follow-up.

(Rev Med Chile 2019; 147: 243-246)

Key words: Cardiac Surgical Procedures; Heart Neoplasms; Heart Valve Disease; Infarction, Middle Cerebral Artery.
'Centro Cardiovascular, Hospita Clínico Regional de Concepción "Dr. Guillermo Grant Benavente". Concepción, Chile.

${ }^{2}$ Departamento de Cirugía, Facultad de Medicina,

Universidad de Concepción.

Concepción, Chile.

Los autores declaran no tener conflictos de interés.

Trabajo no recibió apoyo financiero.

Recibido el 9 de agosto de 2018, aceptado el 10 de enero de 2019.

Correspondencia a:

Dr. Roberto González Lagos Departamento de Cirugía

Facultad de Medicina, Universidad de Concepción, Janequeo esquina Chacabuco S/N, Concepción, Chile. rgonzalezlagos@udec.cl

\section{I}

os accidentes cerebrovasculares (ACV) isquémicos de tipo embólicos tienen como origen infrecuente los tumores cardíacos, sin embargo, dentro del estudio ecocardiográfico de la causa embólica central pueden encontrarse como posible etiología $a^{1,2}$.

Los tumores cardíacos primarios son infrecuentes, de estos, el $75 \%$ son benignos, siendo el mixoma el más común, alcanzando más de la mitad de los casos ${ }^{3}$. El fibroelastoma papilar (FP) corresponde a $1-2 \%$ de los tumores cardíacos primarios y se ubica la mayoría de las veces sobre las válvulas cardíacas, siendo la valvula aórtica el lugar más frecuente ${ }^{4,5}$.

Habitualmente, los tumores cardíacos son asintomáticos y constituyen un hallazgo, ya sea, en el examen ecocardiográfico, en el intraoperatorio de cirugía cardicaca o post mortem ${ }^{6}$. El FP no es la excepción, sin embargo, los ubicados sobre las válvulas mitral y aórtica producen sintomatología de manera más frecuente. Las manifestaciones clínicas de los tumores cardíacos dependen de la ubicación y el tamaño del tumor, presentándose generalmente con embolías o con síntomas y signos de insuficiencia cardíaca.

Se presenta un caso clínico de un paciente joven con accidente vascular encefálico isquémico embólico, que mediante ecocardiografía en busca de fuente cardioembólica se pesquisa un tumor cardíaco valvular. 


\section{Presentación del caso}

Paciente de 29 años de edad, sexo masculino, sin antecedentes mórbidos de interés, asintomático desde el punto de vista cardiológico, consultó en el Servicio de Urgencia por episodio súbito de afasia motora y hemiparesia derecha. Al examen físico se encontró sin deterioro hemodinámico y sin otra focalidad neurológica. Se realizó una tomografía computada (TC) de encéfalo que mostró un infarto cerebral correspondiente al territorio de la arteria cerebral media izquierda. Ecotomografía Doppler carotídea y angiotomografía axial computada de vasos del cuello descartaron enfermedad aterosclerótica y disección arterial. Dado el perfil embolígeno del paciente, se realizó una ecocardiografía transtorácica, que mostró una masa ovoídea de $8 \times 7 \times 7 \mathrm{~mm}$ en relación al aparato subvalvular de la zona A2 de la válvula mitral y descartó otros defectos intracardiacos (Figura 1A). Se inició tratamiento anticoagulante $y$, una vez estabilizado desde el punto de vista neurológico, se decidió la exéresis de la lesión cardíaca.

Bajo anestesia general, se abordó por esteronomía media y se conectó a circulación extracorpórea. Se realizó un abordaje transeptal y se exploró la válvula mitral, encontrándose un tumor macroscópicamente compatible con un fibroelastoma papilar ovillado en las cuerdas tendíneas del aparato subvalvular mitral a nivel de la zona A2 (Figura 1B). El tumor se desplegó y se resecó desde su base, preservándose indemne el velo mitral anterior. La pieza quirúrgica fue desplegada en agua, mostrando la macroscopía típica del FA descrita como una anémona marina (Figura 1C). La ecocardiografía intraoperatoria mostró resección completa y una válvula mitral sin disfunción.

El estudio histopatológico de la pieza mostró un tumor con proyecciones papilares formadas por tejido conectivo escasamente celular, sin componente vascular ni inflamatorio, tapizado por una hilera única de células endoteliales, confirmando

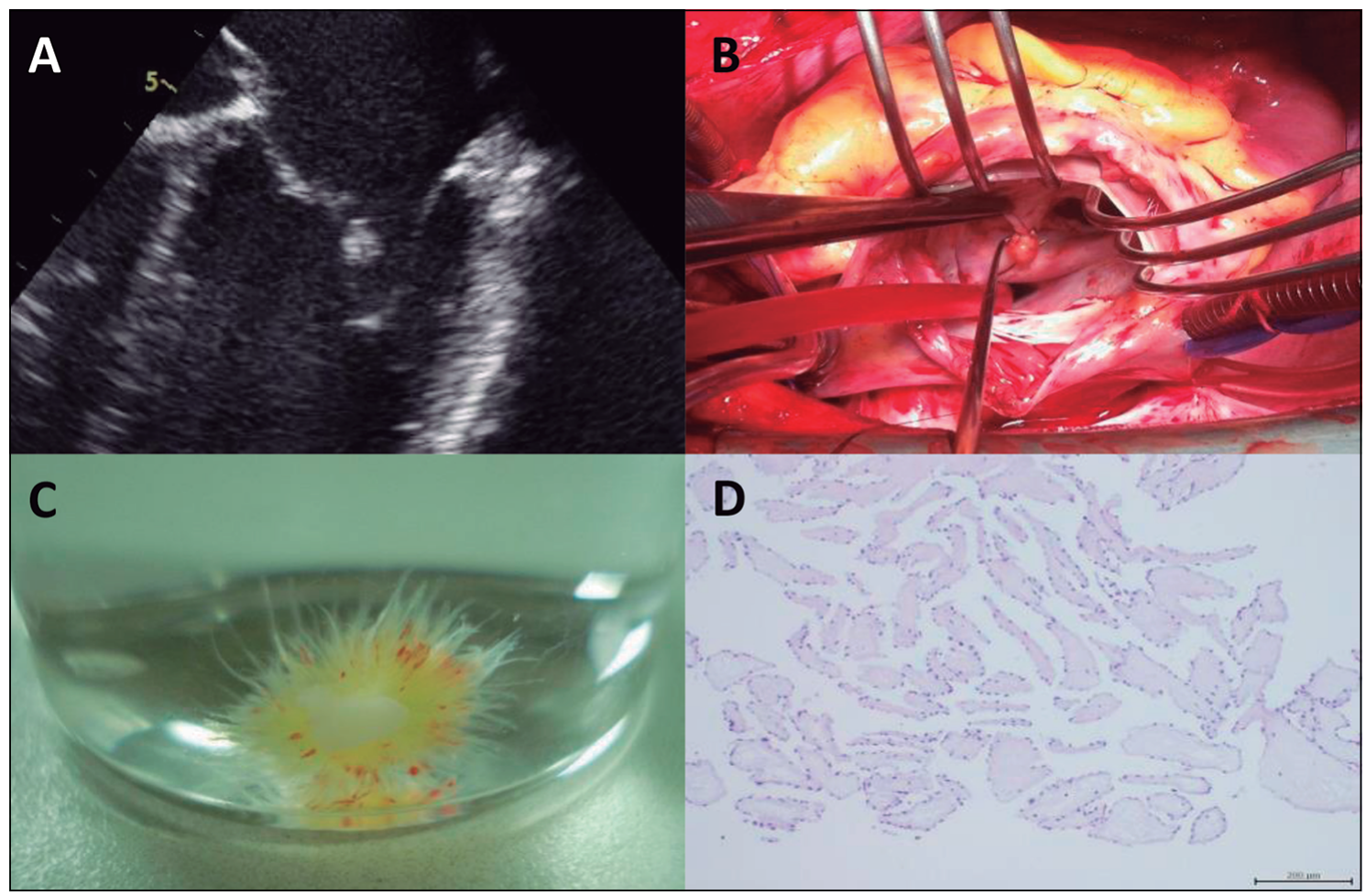

Figura 1. A) Ecocardiografía de superficie que muestra masa ovoídea en relación al aparato subvalvular de la zona A2 de la válvula mitral; B) Fibroelastoma papilar ovillado en las cuerdas tendíneas del aparato subvalvular mitral; C) Fibroelastoma papilar en medio líquido; D) Microscopía: lesión de arquitectura papilar, con centro conectivo escasamente celular y tapizamiento endotelial, compatible con un fibroelastoma papilar. 
el diagnóstico intraoperatorio de fibroelastoma papilar de válvula mitral (Figura 1D).

El paciente no presentó complicaciones en el postoperatorio y fue dado de alta hospitalaria al quinto día. En control ecocardiográfico sin disfunción valvular. A 5 años de seguimiento se encuentra sin síntomas cardiovasculares y con afasia de expresión leve.

\section{Discusión}

Los tumores cardíacos son infrecuentes, su prevalencia es de 0,0017 a $0,28 \%$ en algunas series de autopsias 7 . Generalmente, se describe que $90 \%$ son secundarios, el 10\% restante está constituido por tumores primarios. Sin embargo, los tumores cardiacos secundarios muchas veces no se diagnostican ni tratan, debido a que se presentan como metástasis en pacientes con cáncer avanzado ${ }^{8}$.

El FP es un tumor benigno originado en el endocardio y representa entre 1 y $2 \%$ de los tumores cardiacos primarios. Se han reportado en distintos grupos etarios, desde neonatos hasta pacientes de la tercera edad, sin embargo, la edad promedio al momento del diagnóstico es de aproximadamente 60 años $^{9}$. Es característica su presencia en la superficie de las válvulas cardíacas, siendo algo más frecuente en la válvula aórtica, seguida por la mitral. En general, los FP de las válvular semilunares pueden ubicarse con igual frecuencia en la cara ventricular o arterial, pero los de las válvulas aurículoventriculares suelen ubicarse hacia la cara auricular ${ }^{10}$. El compromiso del aparato subvalvular mitral es raro y solo ha sido reportado en algunos $\operatorname{casos}^{11}$.

El método de elección para el diagnóstico es la ecocardiografía (transtorácica, transesofágica o ambas), que permite caracterizar la morfología, ubicación y movilidad del tumor, así como la determinación de sus consecuencias hemodinámicas. La tomografía computada multicorte y la resonancia magnética cardíaca son técnicas complementarias y útiles en el caso de hallazgos poco concluyentes en la ecocardiografía ${ }^{12}$. El diagnóstico diferencial se debe realizar con otros tumores cardiacos, vegetaciones y trombos intracardiacos, sobre todo cuando se ubican en cavidades derechas, paredes auriculares o cuerdas tendíneas de válvulas aurículoventriculares ${ }^{13}$.

Este tipo de tumor cardíaco ofrece una gran variedad de presentaciones clínicas. La mayoría son asintomáticos, siendo hallazgos de autopsias. Los FP del lado derecho suelen ser asintomáticos y rara vez se presentan como embolía pulmonar, mientras que los de ubicación izquierda tienen riesgo de embolización sistémica, siendo más frecuente la embolía cerebral ${ }^{14,15}$. En los FP ubicados en la válvula mitral el riesgo embólico es mayor que entre los ubicados en la válvula aórtica9 . La embolía se puede producir debido al desprendimiento de fragmentos tumorales o de trombos formados en relación al tumor ${ }^{16}$. Sin embargo, también se han reportado casos que presentan síntomas de insuficiencia cardíaca, muerte súbita o síndrome coronario ${ }^{17}$.

El tratamiento recomendado en los casos sintomáticos es la extirpación quirúrgica. En pacientes asintomáticos se debe tomar en consideración la ubicación, tamaño y movilidad tumoral. En aquellos pacientes asintomáticos con FP del lado izquierdo, móviles y mayores de $1 \mathrm{~cm}$ también se recomienda el tratamiento quirúrgico ${ }^{18}$. La cirugía es un tratamiento curativo, sin existir reportes de recidiva tumoral.

\section{Referencias}

1. Abbasi AS, Da Costa M, Hennessy T, Kiernan TJ. Cardiac papillary fibroelastoma presenting as acute stroke. BMJ Case Rep 2013; 12: 2013.

2. Caballero J, Calle G, Arana R, Sancho M, Caballero FJ, Pinero C, et al. Fibroelastoma Papilar Cardíaco. Diferentes Formas de Presentación Clínica. Rev Esp Cardiol 1997; 50: 815-7.

3. González R, Raffo M, Alarcón E, Ghyra A, Saldías R, Stockins A, et al. Tumores Cardíacos. Rev Chil Cir 2005; 57: 195-8.

4. Roa J, Viedma A, Martínez F, Martínez B. AVC isquémico embolígeno secundario a fibroelastoma papilar cardíaco. Cardiocore 2010; 45: 36-8.

5. Mohammadi S, Martineau A, Voisine P, Dagenais F. Left atrial papillary fibroelastoma: a rare cause of multiple cerebral emboli. Ann Thorac Surg 2007; 84: 1396-7.

6. Boodhwani M, Veinot JP, Hendry PJ. Surgical approach to cardiac papillary fibroelastomas. Can J Cardiol 2007; 23: 301-2.

7. Reynen K. Frequency of primary tumors of the heart. Am J Cardiol 1996; 77: 107.

8. Arnaiz P, Toledo I, Borzutzky A, Urcelay G, Heusser F, Garay F, et al. Comportamiento clínico de los tumores 
cardíacos desde el feto hasta el adulto: serie multicéntrica de 38 pacientes. Rev Med Chile 2006; 134 (9): 113545.

9. Gowda RM, Khan IA, Nair CK, Mehta NJ, Vasavada BC, Sacchi TJ. Cardiac papillary fibroelastoma: a comprehensive analysis of 725 cases, Am Heart J 2003; 146: 404-10.

10. Shahian DM, Labib SB, Chang G. Cardiac papillary fibroelastoma. Ann Thorac Surg 1995; 59: 538-41.

11. Ziabakhsh S, Jalalian R, Mokhtari-Esbuie F. Papillary fibroelastoma of a mitral valve chordae, presenting with atypical chest pain and palpitation: A case report and the literature Caspian J Intern Med 2014; 5 (2): 123-6.

12. Bruce C. Cardiac tumours: diagnosis and management. Heart 2011; 97: 151-60.

13. Córdova S, Zalaquett R, Chuaqui B, Casanegra P, Chamorro G. Fibroelastoma papilar múltiple de válvula aórtica complicado de accidente vascular encefálico embólico: Caso clínico. Rev Med Chile 2001; 129 (9):
1056-60.

14. Al-Mohammad A, Pambakian H, Young C. Fibroelastoma: case report and review of the literature. Heart 1998; 79: 301-4.

15. Tamin SS, Maleszewski JJ, Scott CG, Khan SK, Edwards WD, Bruce CJ, et al. Prognostic and Bioepidemiologic Implications of Papillary Fibroelastomas. J Am Coll Cardiol 2015; 65 (22): 2420-9.

16. Fowles R, Miller D, Egbert B, Fitzgerald J, Popp R. Systemic embolization from a mitral valve papillary endocardial fibroma detected by two-dimensional echocardiography. Am Heart J 1981; 102: 128-30.

17. Liebeskind DS, Buljubasic N, Saver JL. Cardioembolic stroke due to papillary fibroelastoma. J Stroke Cerebrovasc Dis 2001; 10: 94-5.

18. Parthenakis F, Nyktari E, Patrianakos A, Pitsis A, Asimaki A, Vardas P. Asymptomatic papillary fibroelastoma of the aortic valve in a young woman-a case report. Cardiovasc Ultrasound 2009; 7: 43. 